PROCEEDINGS OF THE

AMERICAN MATHEMATICAL SOCIETY

Volume 138, Number 12, December 2010, Pages 4205-4216

S 0002-9939(2010)10456-2

Article electronically published on June 30, 2010

\title{
INDISPENSABLE BINOMIALS IN SEMIGROUP IDEALS
}

\author{
IGNACIO OJEDA AND ALBERTO VIGNERON-TENORIO
}

(Communicated by Bernd Ulrich)

\begin{abstract}
In this paper, we deal with the problem of the uniqueness of a minimal system of binomial generators of a semigroup ideal. Concretely, we give different necessary and/or sufficient conditions for the uniqueness of such a minimal system of generators. These conditions come from the study and combinatorial description of the so-called indispensable binomials in the semigroup ideal.
\end{abstract}

\section{INTRODUCTION}

Under suitable conditions on a finitely generated semigroup $\mathcal{A}=\mathbf{a}_{1} \mathbb{N}+\ldots+\mathbf{a}_{r} \mathbb{N}$, a binomial ideal $I_{\mathcal{A}}$ of the polynomial ring in $r$ variables over a field $\mathbb{k}, R=$ $\mathbb{k}\left[X_{1}, \ldots, X_{r}\right]$, is determined: the so-called semigroup ideal of $\mathcal{A}$ (see Section 1 for the details).

Semigroup ideals play a relevant role in (Computational) Commutative Algebra and Algebraic Geometry. Moreover, they have a lot of applications in different areas such as Statistics, Operational Research or Phylogenetic reconstruction (to cite only three examples).

As in the case of monomial ideals, semigroup ideals have a rich combinatorial structure coming directly from the semigroup (see, e.g., [3, 6] and [15]). However, although reasonable conditions on $\mathcal{A}$ guarantee the existence of minimal systems of binomial generators for $I_{\mathcal{A}}$, they need not be unique, in contrast with the monomial case.

Many successful efforts have been made to compute and describe (minimal) systems of binomial generators of semigroup ideals (see, e.g., 4] or Chapter 9 in [21] and the references therein), but only recently has the problem of the uniqueness been explicitly treated. In fact, the interest in this problem arises first from Algebraic Statistics ([23]), in which the systems of binomial generators of particular families of semigroup ideals defining certain statistics models are called Markov bases of the model ([7]). Subsequently, different authors have investigated this uniqueness problem. In [1] and in [14, the notions of indispensable binomial and monomial are introduced, respectively, and in [5] both properties are studied from

Received by the editors October 23, 2009 and, in revised form, February 22, 2010.

2010 Mathematics Subject Classification. Primary 13F20; Secondary 16W50, 13 F55.

Key words and phrases. Semigroup ideal, indispensable binomial, minimal system of generators, Markov basis, simplicial complex, toric ideal, monomial algebra.

Both authors are partially supported by the project MTM2007-64704, National Plan I+D+I. The first author is partially supported by Junta de Extremadura (ayuda a grupos GRU09104) and FEDER funds.

(C) 2010 American Mathematical Society Reverts to public domain 28 years from publication 
a combinatorial point of view. It is convenient to observe that one can find in the literature results which imply a characterization of particular families of semigroup ideals with a unique system of binomial generators (see Corollary 3.4 in [18], Corollary 3.11 in [16] or Proposition 1 in [20]). Furthermore, it is known that the generic lattice ideals introduced by I. Peeva and B. Sturmfels in [18, have a unique minimal system of binomial generators (see Lemma 3.3 and Remark 4.4.3 in [18]); therefore, one may assume that there exist semigroup ideals with arbitrary large systems of indispensable binomials generators (see [17). All these facts suggest that the uniqueness problem might be connected with other interesting problems about semigroup ideals.

Recall that a binomial in a semigroup ideal $I_{\mathcal{A}}$ is said to be indispensable if it belongs (up to a scalar multiple) to every system of binomial generators $I_{\mathcal{A}}$ (see [13]). Thus, $I_{\mathcal{A}}$ has a unique system of binomial generators if, and only if, it is generated by indispensable binomials. On the other hand, a monomial is called indispensable if it appears (up to a scalar multiple) in, at least, one binomial of any system of binomial generators of $I_{\mathcal{A}}$.

Indispensable monomials were introduced by Aoki, Takemura and Yoshida in [1]. They are considered as a first approximation to the study of indispensable binomials; notice that any indispensable binomial is a difference of two indispensable monomials. Moreover, indispensable monomials always exist (see Proposition 3.1 in [5]) in clear contrast to the indispensable binomials (consider, e.g., $I_{\mathcal{A}}=\langle x-y, x-z\rangle$ ).

In the first part of this paper (Section 2), we give a combinatorial necessary and sufficient condition for the existence of indispensable binomials in a semigroup ideal (Theorem 8). Our condition depends on the knowledge of some simplicial complexes associated to the semigroup introduced by S. Eliahou in his $\mathrm{PhD}$ Thesis (9]). As a consequence, an explicit characterization of all indispensable binomials and monomials of a semigroup ideal $I_{\mathcal{A}}$ is given. In Section 3 , we deal with the problem of the existence of indispensable binomials in a semigroup ideal $I_{\mathcal{A}}$ by using only Gröbner bases techniques; concretely, we give an effective necessary and sufficient condition for the existence of indispensable binomials in $I_{\mathcal{A}}$ consisting of the computation of $r$ (the number of indeterminates of the corresponding polynomial ring) Gröbner bases (Theorem 13). We end the paper by illustrating our results with an example borrowed from Algebraic Statistics.

\section{Preliminaries: Definitions and notation}

Let $\mathcal{A}$ be a commutative semigroup with zero element $0 \in \mathcal{A}$ and let $G(\mathcal{A})$ be a commutative group with a semigroup homomorphism $\iota: \mathcal{A} \rightarrow G(\mathcal{A})$ such that every homomorphism from $\mathcal{A}$ to a group factors in a unique way through $\iota$. The commutative group $G(\mathcal{A})$ exists and is unique up to isomorphism; it is called the associated commutative group of $\mathcal{A}$. Further, $G(\mathcal{A})$ is finitely generated when $\mathcal{A}$ is. The map $\iota$ is injective if, and only if, $\mathcal{A}$ is cancellative, that is to say, if $\mathbf{a}+\mathbf{b}=\mathbf{a}+\mathbf{c}, \mathbf{a}, \mathbf{b}, \mathbf{c} \in \mathcal{A}$, then $\mathbf{b}=\mathbf{c}$; in this case, $G(\mathcal{A})$ is the smallest group containing $\mathcal{A}$.

Throughout this paper, $\mathcal{A}$ will denote a finitely generated commutative cancellative semigroup with zero element. Moreover, we will assume always that $\mathcal{A}$ is combinatorially finite; that is to say, there are only finitely many ways to write $\mathbf{a} \in \mathcal{A} \backslash\{0\}$ as a sum $\mathbf{a}=\mathbf{a}_{1}+\ldots+\mathbf{a}_{q}$, with $\mathbf{a}_{i} \in \mathcal{A} \backslash\{0\}$. Equivalently, $\mathcal{A}$ is combinatorially finite if, and only if, $\mathcal{A} \cap(-\mathcal{A})=\{0\}$ (see Proposition 1.1 in [4]). 
Notice that this property guarantees that $\mathbf{b} \preceq \mathcal{A} \mathbf{a} \Longleftrightarrow \mathbf{a}-\mathbf{b} \in \mathcal{A}$ is a well defined partial order on $\mathcal{A}$.

Examples of finitely generated commutative cancellative combinatorially finite semigroups with zero element (semigroups in the following) are the subsemigroup of $\mathbb{N}^{d}$ generated by the columns of a non-negative integer matrix $A \in \mathbb{N}^{d \times r}$.

Let $\mathbb{k}$ be a fixed arbitrary field. We write $\mathbb{k}[\mathcal{A}]$ for the $\mathbb{k}$-vector space

$$
\mathbb{k}[\mathcal{A}]=\bigoplus_{\mathbf{a} \in \mathcal{A}} k \mathbf{t}^{\mathbf{a}}
$$

endowed with a multiplication which is $\mathbb{k}$-linear and such that $\mathbf{t}^{\mathbf{a}} \cdot \mathbf{t}^{\mathbf{b}}:=\mathbf{t}^{\mathbf{a}+\mathbf{b}}, \mathbf{a}, \mathbf{b} \in$ $\mathcal{A}$. Thus $\mathbb{k}[\mathcal{A}]$ has a natural $\mathbb{k}$-algebra structure, and we will refer to it as the semigroup algebra of $\mathcal{A}$.

The choice of a system of generators $\mathbf{a}_{1}, \ldots, \mathbf{a}_{r}$ of $\mathcal{A}$ induces a natural $\mathcal{A}$-grading on $R:=\mathbb{k}\left[X_{1}, \ldots, X_{r}\right]$, by assigning weight $\mathbf{a}_{i}$ to $X_{i}, i=1, \ldots, r$; that is to say,

$$
R=\bigoplus_{\mathbf{a} \in \mathcal{A}} R_{\mathbf{a}}
$$

where $R_{\mathbf{a}}$ is the vector subspace of $R$ generated by all the monomials $X^{\mathbf{u}}:=$ $X_{1}^{u_{1}} \cdots X_{r}^{u_{r}}$ with $\sum_{i=1}^{r} u_{i} \mathbf{a}_{i}=\mathbf{a}$ and $\mathbf{u}=\left(u_{1}, \ldots, u_{r}\right)^{\prime} \in \mathbb{N}^{r}$ (the prime means transpose). Since $\mathcal{A}$ is combinatorially finite, the vector spaces $R_{\mathrm{a}}$ are finite dimensional (see Proposition 1.2 in [4]). Concretely, there are $\operatorname{dim}_{\mathrm{k}} R_{\mathbf{a}}$ monomials of $\mathcal{A}$-degree $\mathbf{a}$, for each $\mathbf{a} \in \mathcal{A}$. Let $M_{\mathbf{a}}$ denote the set of monomials in $R_{\mathbf{a}}$.

In the following, we will assume that $\mathbf{a}_{1}, \ldots, \mathbf{a}_{r}$ is a fixed system of generators of $\mathcal{A}$.

The natural semigroup morphism $\pi: \mathbb{N}^{r} \rightarrow \mathcal{A} ; \mathbf{u} \mapsto \sum_{i=1}^{r} u_{i} \mathbf{a}_{i}$ defines an $\mathcal{A}$ (multi)graded surjective $\mathbb{k}$-algebra morphism

$$
\varphi_{0}: R=\mathbb{k}\left[\mathbb{N}^{r}\right] \longrightarrow \mathbb{k}[\mathcal{A}] ; X_{i} \longmapsto \mathbf{t}^{\mathbf{a}_{i}} .
$$

Thus, the ideal $I_{\mathcal{A}}:=\operatorname{ker}\left(\varphi_{0}\right)$ is an $\mathcal{A}$-homogeneous ideal called the (semigroup) ideal of $\mathcal{A}$. Notice that $I_{\mathcal{A}}$ is a toric ideal when $G(\mathcal{A})$ is torsion free (see [22], Chapter 4).

It is well known (see [11]) that $I_{\mathcal{A}}$ is the ideal of $R$ generated by

$$
\left\{X^{\mathbf{u}}-X^{\mathbf{v}}: \sum_{i=1}^{r} u_{i} \mathbf{a}_{i}=\sum_{i=1}^{r} v_{i} \mathbf{a}_{i}\right\}
$$

where $X^{\mathbf{u}}=X_{1}^{u_{1}} \cdots X_{r}^{u_{r}}$ and $X^{\mathbf{v}}=X_{1}^{v_{1}} \cdots X_{r}^{v_{r}}$, as usual. Therefore, there exist minimal systems of $\mathcal{A}$-homogeneous generators of $I_{\mathcal{A}}$ consisting of finitely many pure difference binomials, i.e. differences of two monomials of the same $\mathcal{A}$-degree (see, e.g. 4, Section 2). In fact, one has the following:

Lemma 1. Every system of binomial generators of $I_{\mathcal{A}}$ is $\mathcal{A}$-graded

Proof. By definition $I_{\mathcal{A}}=\operatorname{ker}\left(\varphi_{0}\right)$. So, if $X^{\mathbf{u}}-\lambda X^{\mathbf{v}} \in I_{\mathcal{A}}$, then

$$
\mathbf{t}^{\sum_{i=1}^{r} u_{i} \mathbf{a}_{i}}=\lambda \mathbf{t}^{\sum_{i=1}^{r} v_{i} \mathbf{a}_{i}} ;
$$

that is to say, $\sum_{i=1}^{r} u_{i} \mathbf{a}_{i}=\sum_{i=1}^{r} v_{i} \mathbf{a}_{i}$ and $\lambda=1$.

The $\mathcal{A}$-degrees of the polynomials appearing in any minimal system of $\mathcal{A}$ homogeneous generators of $I_{\mathcal{A}}$ do not depend on the system of generators: it is well known that the number of polynomials of $\mathcal{A}$-degree $\mathbf{a} \in \mathcal{A}$ in a minimal system of $\mathcal{A}$-homogeneous generators is $\operatorname{dim}_{k} \operatorname{Tor}_{1}^{R}(\mathbb{k}, \mathbb{k}[\mathcal{A}])_{\mathbf{a}}$ (see, e.g. [12], 
Section 8.3). Thus, we say that $I_{\mathcal{A}}$ has minimal generators in degree a when $\operatorname{dim}_{\mathfrak{k}} \operatorname{Tor}_{1}^{R}(\mathbb{k}, \mathbb{k}[\mathcal{A}])_{\mathbf{a}} \neq 0$.

Definition 2. We say that $\mathbf{a} \in \mathcal{A}$ is a minimal $\mathcal{A}$-degree of $I_{\mathcal{A}}$ if $I_{\mathcal{A}}$ has minimal generators in degree $\mathbf{a}$.

Note that there are finitely many minimal $\mathcal{A}$-degrees of $I_{\mathcal{A}}$.

Definition 3. We say that $\mathbf{a} \in \mathcal{A}$ is an indispensable $\mathcal{A}$-degree of $I_{\mathcal{A}}$ if every (minimal) system of $\mathcal{A}$-homogeneous generators of $I_{\mathcal{A}}$ contains one, and only one, polynomial of $\mathcal{A}$-degree a. In this case, such a polynomial is a binomial and it is said to be an indispensable binomial of $I_{\mathcal{A}}$.

Notice first that every indispensable $\mathcal{A}$-degree is a minimal $\mathcal{A}$-degree. Furthermore, observe that an indispensable binomial of $I_{\mathcal{A}}$ appears (up to a scalar multiple) in every system of $\mathcal{A}$-homogeneous generators of $I_{\mathcal{A}}$. Moreover, it is easy to see that a binomial that appears (up to a scalar multiple) in every system of $\mathcal{A}$-homogeneous generators of $I_{\mathcal{A}}$ is indispensable. Indeed, if $\mathcal{S}$ is a system of $\mathcal{A}$ homogeneous generators of $I_{\mathcal{A}}$ containing two different polynomials, $f$ and $g$, of the same $\mathcal{A}$-degree, by substituting $f$ by $f-g$ in $\mathcal{S}$, we obtain a new system of $\mathcal{A}$-homogeneous generators of $I_{\mathcal{A}}$ not containing $f$. That is to say, if $f \in I_{\mathcal{A}}$ is not indispensable, it does not belong to every system of $\mathcal{A}$-homogeneous generators of $I_{\mathcal{A}}$

Summarizing, we have:

Proposition 4. The following statements are equivalent:

(a) $I_{\mathcal{A}}$ has a unique (up to a scalar multiple) minimal system of $\mathcal{A}$-homogeneous generators.

(b) Every minimal system of $\mathcal{A}$-homogeneous generators consists of binomials.

(c) $I_{\mathcal{A}}$ has a unique minimal system of (pure difference) binomial generators.

(d) $I_{\mathcal{A}}$ is generated by its indispensable binomials.

\section{COMbinatorial DESCRIPTION OF INDISPENSABILITY}

In this section, we will give a necessary and sufficient condition for the existence of indispensable binomials in $I_{\mathcal{A}}$ for a given semigroup $\mathcal{A}=\mathbf{a}_{1} \mathbb{N}+\ldots+\mathbf{a}_{r} \mathbb{N}$.

We start by introducing a combinatorial object associated to $\mathcal{A}$.

Definition 5. For any $\mathbf{a} \in \mathcal{A}$, define the abstract simplicial complex $\nabla_{\mathbf{a}}$ on the vertex set $M_{\mathbf{a}}=\left\{X^{\mathbf{u}}=X_{1}^{u_{1}} \cdots X^{u_{r}} \mid \sum_{i=1}^{r} u_{i} \mathbf{a}_{i}=\mathbf{a}\right\}$

$$
\nabla_{\mathbf{a}}=\left\{F \subseteq M_{\mathbf{a}} \mid \operatorname{gcd}(F) \neq 1\right\},
$$

where $\operatorname{gcd}(F)$ denotes the greatest common divisor of the monomials in $F$.

Recall that $M_{\mathbf{a}}$ has finitely many monomials because of the combinatorial finiteness of $\mathcal{A}$. So, the simplicial complexes $\nabla_{\mathbf{a}}$ are finite.

The next proposition was first proved by S. Eliahou, who introduced the simplicial complexes $\nabla_{\mathbf{a}}$ in $[9$. Other proofs, in a more general context, can be found in [15] and in [6].

Proposition 6. Let $\mathbf{a} \in \mathcal{A}$. a is a minimal $\mathcal{A}$-degree of $I_{\mathcal{A}}$ if, and only if, $\nabla_{\mathbf{a}}$ is not connected. 
It is important to observe that the 1 -skeleton of $\nabla_{\mathbf{a}}$ is a subgraph of the graph given in [5] (Definition 2.1) with the same set of vertices and the same number of connected components. Thus, one can use the simplicial complexes $\nabla_{\mathbf{a}}$ to obtain the same results as in that paper. In fact, Theorems 2.6 and 2.7 in 5 ] can be understood as a new version of Theorem 2.5 in 4 for the simplicial complexes $\nabla_{\mathbf{a}}$ by taking into account that both the simplicial complexes in 4 and the simplicial complexes $\nabla_{\mathbf{a}}$ have isomorphic homology $\mathbb{k}$-vector spaces (see [15], Theorem 3).

An immediate consequence of Proposition 6 is the following:

Corollary 7. Let $\mathbf{a} \in \mathcal{A}$. Then $\mathbf{a}$ is an indispensable $\mathcal{A}$-degree of $I_{\mathcal{A}}$ if, and only if, $\nabla_{\mathbf{a}}=\left\{\left\{X^{\mathbf{u}}\right\},\left\{X^{\mathbf{v}}\right\}\right\}$.

Proof. Let a be an indispensable $\mathcal{A}$-degree of $I_{\mathcal{A}}$ and let $X^{\mathbf{u}}-X^{\mathbf{v}} \in I_{\mathcal{A}}$ be the corresponding indispensable binomial. If there exists a monomial $X^{\mathbf{w}} \in M_{\mathbf{a}}$ different from $X^{\mathbf{u}}$ and $X^{\mathbf{v}}$, we could replace $X^{\mathbf{u}}-X^{\mathbf{v}}$ by $X^{\mathbf{u}}-X^{\mathbf{w}}$ and $X^{\mathbf{v}}-X^{\mathbf{w}}$ in a (minimal) system of generators of $I_{\mathcal{A}}$, thus obtaining a (not-necessarily minimal) system of generators of $I_{\mathcal{A}}$ not containing $X^{\mathbf{u}}-X^{\mathbf{v}}$, which is not possible by Definition 3. Therefore, $M_{\mathbf{a}}=\left\{X^{\mathbf{u}}, X^{\mathbf{v}}\right\}$ and, by Proposition 6, we conclude that $\nabla_{\mathbf{a}}=\left\{\left\{X^{\mathbf{u}}\right\},\left\{X^{\mathbf{v}}\right\}\right\}$, i.e., gcd $\left(X^{\mathbf{u}}, X^{\mathbf{v}}\right)=1$ (recall that every indispensable $\mathcal{A}$-degree is a minimal $\mathcal{A}$-degree).

Conversely, if $\nabla_{\mathbf{a}}=\left\{\left\{X^{\mathbf{u}}\right\},\left\{X^{\mathbf{v}}\right\}\right\}$, by Proposition 6] a is a minimal $\mathcal{A}$-degree of $I_{\mathcal{A}}$. Moreover, since the only polynomial of $\mathcal{A}$-degree (up to a scalar multiple) is $X^{\mathbf{u}}-X^{\mathbf{v}}$, we conclude that it has to be indispensable and so $\mathbf{a}$ is an indispensable $\mathcal{A}$-degree of $I_{\mathcal{A}}$.

The above result was also noticed by H. Charalambous et al. (see Theorem 4.1 in $[5])$.

Theorem 8. Let $\mathbf{a} \in \mathcal{A}$. Then $\mathbf{b} \prec_{\mathcal{A}} \mathbf{a}$ is indispensable if, and only if, there exists $\left\{X^{\mathbf{u}}, X^{\mathbf{v}}\right\} \in \nabla_{\mathbf{a}}$ such that

(a) $\operatorname{gcd}\left(X^{\mathbf{u}}, X^{\mathbf{v}}\right) \neq \operatorname{gcd}\left(X^{\mathbf{u}}, X^{\mathbf{v}}, X^{\mathbf{w}}\right)$, for every 2 -dimensional face $\left\{X^{\mathbf{u}}, X^{\mathbf{v}}\right.$, $\left.X^{\mathbf{w}}\right\} \in \nabla_{\mathbf{a}}$

(b) $\operatorname{gcd}\left(X^{\mathbf{u}}, X^{\mathbf{v}}\right)$ has $\mathcal{A}$-degree $\mathbf{a}-\mathbf{b}$.

In this case,

$$
\operatorname{gcd}\left(X^{\mathbf{u}}, X^{\mathbf{v}}\right)^{-1}\left(X^{\mathbf{u}}-X^{\mathbf{v}}\right)
$$

is the corresponding indispensable binomial of $I_{\mathcal{A}}$.

Proof. If $\mathbf{b} \prec_{\mathcal{A}} \mathbf{a}$ is an indispensable $\mathcal{A}$-degree of $I_{\mathcal{A}}$, then, by Corollary $7, \nabla_{\mathbf{b}}=$ $\left\{\left\{X^{\overline{\mathbf{u}}}\right\},\left\{X^{\overline{\mathbf{v}}}\right\}\right\}$. Let $X^{\mathbf{z}}$ be a monomial of $\mathcal{A}$-degree $\mathbf{a}-\mathbf{b} \neq 0$ and consider $X^{\mathbf{u}}=$ $X^{\overline{\mathbf{u}}+\mathbf{z}}$ and $X^{\mathbf{v}}=X^{\overline{\mathbf{v}}+\mathbf{z}}$. Notice that $\left\{X^{\mathbf{u}}, X^{\mathbf{v}}\right\} \in \nabla_{\mathbf{a}}$ because $\operatorname{gcd}\left(X^{\mathbf{u}}, X^{\mathbf{v}}\right)=X^{\mathbf{z}} \neq$ 1. If there exists $X^{\mathbf{w}} \in M_{\mathbf{a}} \backslash\left\{X^{\mathbf{u}}, X^{\mathbf{v}}\right\}$ with $\operatorname{gcd}\left(X^{\mathbf{u}}, X^{\mathbf{v}}, X^{\mathbf{w}}\right)=\operatorname{gcd}\left(X^{\mathbf{u}}, X^{\mathbf{v}}\right)$, then $X^{\mathbf{z}}$ divides $X^{\mathbf{w}}$ and so $X^{\mathbf{w}} / X^{\mathbf{z}} \in \nabla_{\mathbf{b}}$.

Conversely, let $\left\{X^{\mathbf{u}}, X^{\mathbf{v}}\right\} \in \nabla_{\mathbf{a}}$ be such that $\operatorname{gcd}\left(X^{\mathbf{u}}, X^{\mathbf{v}}\right)$ has $\mathcal{A}$-degree $\mathbf{a}-\mathbf{b}$ and $\operatorname{gcd}\left(X^{\mathbf{u}}, X^{\mathbf{v}}\right) \neq \operatorname{gcd}\left(X^{\mathbf{u}}, X^{\mathbf{v}}, X^{\mathbf{w}}\right)$, for every $X^{\mathbf{w}} \in M_{\mathbf{a}} \backslash\left\{X^{\mathbf{u}}, X^{\mathbf{v}}\right\}$. Since $\operatorname{gcd}\left(X^{\mathbf{u}}, X^{\mathbf{v}}\right) \neq 1$, the monomials $X^{\mathbf{u}} / \operatorname{gcd}\left(X^{\mathbf{u}}, X^{\mathbf{v}}\right)$ and $X^{\mathbf{v}} / \operatorname{gcd}\left(X^{\mathbf{u}}, X^{\mathbf{v}}\right)$ have $\mathcal{A}$-degree $\mathbf{b} \prec_{\mathcal{A}} \mathbf{a}$ and $\mathbf{b}$ is indispensable. Otherwise, by Corollary 7 there exists $X^{\overline{\mathbf{w}}} \in \nabla_{\mathbf{b}}$ which is different from the other ones, and $\operatorname{so} \operatorname{gcd}\left(X^{\mathbf{u}}, X^{\mathbf{v}}\right)=\operatorname{gcd}\left(X^{\mathbf{u}}\right.$, $\left.X^{\mathbf{v}}, X^{\mathbf{w}}\right)$, with $X^{\mathbf{w}}=X^{\overline{\mathbf{w}}} \operatorname{gcd}\left(X^{\mathbf{u}}, X^{\mathbf{v}}\right)$. 
Since there exists $\mathbf{a} \in \mathcal{A}$ such that $\mathbf{b} \prec_{\mathcal{A}} \mathbf{a}$ for every minimal $\mathcal{A}$-degree, $\mathbf{b}$, of $I_{\mathcal{A}}$ (see, e.g., [22], Chapter 4), in order to check the existence of indispensable binomials, it suffices to compute one (huge) simplicial complex $\nabla_{\mathbf{a}}$ and then use Theorem 8. Of course, this theoretical assertion is not very practical because these bounds are very coarse. Nevertheless, in some particular cases, one can find tight bounds for the minimal $\mathcal{A}$-degrees of $I_{\mathcal{A}}$, which combined with the high intrinsic symmetry of the simplicial complexes $\nabla_{\mathbf{a}}$ allows us to check the existence of indispensable $\mathcal{A}$-degrees and compute all the indispensable binomials. This combined approach is applied in Section 4

Similar strategies may be used to compute (all) the indispensable monomials of $I_{\mathcal{A}}$

Definition 9. We say that $X^{\mathbf{u}} \in R_{\mathbf{a}}$ is an indispensable monomial of $I_{\mathcal{A}}$ if every system of binomial generators of $I_{\mathcal{A}}$ contains, at least, a binomial (up to a scalar multiple) of the form $X^{\mathbf{u}}-X^{\mathbf{v}}$. In this case, we say that $\mathbf{a}$ is quasi-indispensable $\mathcal{A}$-degree of $I_{\mathcal{A}}$.

Similarly to Corollary 7 we may state the following:

Corollary 10. Let $\mathbf{a} \in \mathcal{A}$. Then $\mathbf{a}$ is quasi-indispensable if, and only if, $\nabla_{\mathbf{a}}$ has, at least, a 0-dimensional connected component and $\pi^{-1}(\mathbf{a})$ has cardinality greater than or equal to 2 .

Notice that the above corollary is nothing but a combinatorial version of Theorem 3.1 in [1].

It is clear that if $X^{\mathbf{u}}-X^{\mathbf{v}}$ is an indispensable binomial of $I_{\mathcal{A}}$, then $X^{\mathbf{u}}$ and $X^{\mathbf{v}}$ are indispensable monomials of $I_{\mathcal{A}}$. Unfortunately, the converse is not true. Nevertheless, in contrast to indispensable binomials, indispensable monomials always exist (see, e.g., Proposition 3.1 in [5]).

By weakening the hypothesis in Theorem 8 , we obtain the following result, which allows us to compute all the indispensable monomials when a sufficiently large $\mathbf{a} \in \mathcal{A}$ is known. We omit its proof because it is quite similar to the proof of Theorem 8 .

Corollary 11. Let $\mathbf{a} \in \mathcal{A}$. For each $X^{\mathbf{u}} \in M_{\mathbf{a}}$ and for each maximal element $\operatorname{gcd}\left(X^{\mathbf{u}}, X^{\mathbf{v}}\right)$ with respect to division in the set $\left\{\operatorname{gcd}\left(X^{\mathbf{u}}, X^{\mathbf{w}}\right) \mid X^{\mathbf{w}} \in M_{\mathbf{a}}\right\}$, the monomial $\operatorname{gcd}\left(X^{\mathbf{u}}, X^{\mathbf{v}}\right)^{-1} X^{\mathbf{u}}$ is an indispensable monomial of $I_{\mathcal{A}}$.

Notice that, unlike Proposition 3.1 in [5], our result does not require the previous computation of a system of generators of $I_{\mathcal{A}}$ to compute all its indispensable monomials.

\section{INDISPENSABILITY AND GRÖBNER BASES}

Let $\mathcal{A}=\mathbf{a}_{1} \mathbb{N}+\ldots+\mathbf{a}_{r} \mathbb{N}$ be a semigroup such that $G(\mathcal{A})$ is torsion free.

In 14 it is shown that a binomial in $I_{\mathcal{A}}$ is indispensable if, and only if, it or its negative belongs to the reduced Gröbner basis of $I_{\mathcal{A}}$ for any lexicographic term order on $R$. In this section, we will prove that it is enough to check this for, at most, $r$ Gröbner basis with respect to a degree reverse lexicographical term order on $R$.

Fix positive integers $d_{1}, \ldots, d_{r}$ such that $I_{\mathcal{A}}$ is homogeneous with respect to the grading $\operatorname{deg}\left(X_{i}\right)=d_{i}$ and this grading is compatible with the $\mathcal{A}$-grading of $I_{\mathcal{A}}$; that is to say, if $X^{\mathbf{u}}$ and $X^{\mathbf{v}}$ have the same $\mathcal{A}$-degree, then $\sum_{i=1}^{r} d_{i} u_{i}=\sum_{i=1}^{r} d_{i} v_{i}$. This is always possible because $\mathcal{A}$ is combinatorially finite and $G(\mathcal{A})$ is torsion free. 
Definition 12. A degree reverse lexicographic term order $\prec$ relative to the above grading on $R$ which has $X_{i}$ as the lowest variable is any term order on $R$ represented by an $r \times r$-matrix whose first row is $\left(d_{1}, \ldots, d_{r}\right)$ and second row is $-\mathbf{e}_{i}^{\prime}$, where $\mathbf{e}_{i}$ is the $i$-th canonical basis vector of $\mathbb{Z}^{r}$.

For a better understanding of the proof of the next result, we recall that the support of a monomial $X^{\mathbf{u}}$ in $R$ is $\operatorname{supp}\left(X^{\mathbf{u}}\right)=\operatorname{supp}(\mathbf{u})=\left\{i \in\{1, \ldots, r\} \mid u_{i} \neq 0\right\}$.

Theorem 13. For each $i \in\{1, \ldots, r\}$, let $\prec_{i}$ be a degree reverse lexicographical term order on $R$ which has $X_{i}$ as the lowest variable. A binomial in $I_{\mathcal{A}}$ is indispensable if, and only if, either it or its negative belongs to the reduced Gröbner basis $\mathcal{G}_{i}$ of $I_{\mathcal{A}}$ with respect to $\prec_{i}, i \in\{1, \ldots, r\}$.

Proof. Suppose that $X^{\mathbf{u}}-X^{\mathbf{v}} \in I_{\mathcal{A}}$ is indispensable. Since any reduced Gröbner basis of $I_{\mathcal{A}}$ consists of binomials (see, e.g., Proposition 1.1 in [8]) and $X^{\mathbf{u}}-X^{\mathbf{v}}$ appears in every system of binomial generators of $I_{\mathcal{A}}$, we may assume that $X^{\mathbf{u}}-$ $X^{\mathbf{v}} \in \mathcal{G}_{i}, i=1, \ldots r$.

Conversely, suppose that $X^{\mathbf{u}}-X^{\mathbf{v}}$ or $X^{\mathbf{v}}-X^{\mathbf{u}}$ belongs to $\mathcal{G}_{i}$, for each $i \in$ $\{1, \ldots, r\}$. First of all, we observe that $\operatorname{gcd}\left(X^{\mathbf{u}}, X^{\mathbf{v}}\right)=1$. Otherwise, $\operatorname{gcd}\left(X^{\mathbf{u}}\right.$, $\left.X^{\mathbf{v}}\right)^{-1}\left(X^{\mathbf{u}}-X^{\mathbf{v}}\right) \in I_{\mathcal{A}}$, and $\operatorname{gcd}\left(X^{\mathbf{u}}, X^{\mathbf{v}}\right)^{-1} X^{\mathbf{u}}$ and $\operatorname{gcd}\left(X^{\mathbf{u}}, X^{\mathbf{v}}\right)^{-1} X^{\mathbf{v}}$ properly divide $X^{\mathbf{u}}$ and $X^{\mathbf{v}}$, respectively. Therefore, neither $X^{\mathbf{u}}-X^{\mathbf{v}}$ nor $X^{\mathbf{v}}-X^{\mathbf{u}}$ belongs to any $\mathcal{G}_{i}$, which is impossible by hypothesis.

Now, suppose that there exists a monomial $X^{\mathbf{w}}$ with the same $\mathcal{A}$-degree as $X^{\mathbf{u}}-X^{\mathbf{v}}$. Let $X^{\overline{\mathbf{u}}}=\operatorname{gcd}\left(X^{\mathbf{u}}, X^{\mathbf{w}}\right)$ and $X^{\overline{\mathbf{v}}}=\operatorname{gcd}\left(X^{\mathbf{v}}, X^{\mathbf{w}}\right)$. So, $X^{\mathbf{w}}=X^{\overline{\mathbf{u}}} X^{\overline{\mathbf{v}}} X^{\overline{\mathbf{w}}}$ with $\operatorname{supp}\left(X^{\overline{\mathbf{u}}}\right) \cap \operatorname{supp}\left(X^{\overline{\mathbf{v}}}\right)=\varnothing$, because $\operatorname{gcd}\left(X^{\mathbf{u}}, X^{\mathbf{v}}\right)=1$. If $X^{\overline{\mathbf{w}}} \neq 1$, we consider $j \in \operatorname{supp}\left(X^{\overline{\mathbf{w}}}\right)$. Then, $X^{\mathbf{w}} \prec_{j} X^{\mathbf{u}}$ and $X^{\mathbf{w}} \prec_{j} X^{\mathbf{v}}$; that is to say, $X^{\mathbf{u}}=\operatorname{in}_{\prec_{j}}\left(X^{\mathbf{u}}-X^{\mathbf{w}}\right)$ and $X^{\mathbf{v}}=\operatorname{in}_{\prec_{j}}\left(X^{\mathbf{v}}-X^{\mathbf{w}}\right)$. So, by the definition of a Gröbner basis, there are two polynomials in $\mathcal{G}_{j}$ whose initial monomials divide $X^{\mathbf{u}}$ and $X^{\mathbf{v}}$, respectively. Therefore, neither $X^{\mathbf{u}}-X^{\mathbf{v}}$ nor $X^{\mathbf{v}}-X^{\mathbf{u}}$ could appear in $\mathcal{G}_{j}$ because of its reducibility. So, we may assume that $X^{\overline{\mathbf{w}}}=1$; that is to say, $X^{\mathbf{w}}=X^{\overline{\mathbf{u}}} X^{\overline{\mathbf{v}}}$. Consider $j \in \operatorname{supp}\left(X^{\overline{\mathbf{v}}}\right)$. We have that $X^{\mathbf{v}} \prec_{j} X^{\mathbf{u}}$. If $X^{\mathbf{w}} \prec_{j} X^{\mathbf{v}}$, then $X^{\mathbf{v}}$ is divisible by the initial monomial with respect to $\prec_{j}$ of some binomial of $I_{\mathcal{A}}$; thus $X^{\mathbf{u}}-X^{\mathbf{v}}$ does not belong in $\mathcal{G}_{j}$. Then $X^{\mathbf{v}} \prec_{j} X^{\mathbf{w}}$ and $X^{\mathbf{v}} / X^{\overline{\mathbf{v}}} \prec_{j} X^{\mathbf{w}} / X^{\overline{\mathbf{v}}}=X^{\overline{\mathbf{u}}}$. In this case, there exists a binomial in $I_{\mathcal{A}}$ whose initial monomial with respect to $\prec_{j}$ properly divides $X^{\mathbf{u}}=\operatorname{in}_{\prec_{j}}\left(X^{\mathbf{u}}-X^{\mathbf{v}}\right)$. So we are in contradiction again.

In conclusion, $\nabla_{\mathbf{a}}=\left\{\left\{X^{\mathbf{u}}\right\},\left\{X^{\mathbf{v}}\right\}\right\}$. Then, by Corollary 7 we may assume that $X^{\mathbf{u}}-X^{\mathbf{v}}$ is an indispensable binomial of $I_{\mathcal{A}}$.

Notice that the above theorem gives an algorithm for computing the indispensable binomials in $I_{\mathcal{A}}$ consisting of the computation of $r$ reduced Gröbner basis. Other algorithms can be found or deduced from the results in [1] and [5]. In the first case, $r$ ! reduced Gröbner basis are needed. In the second case, the indispensable binomials in $I_{\mathcal{A}}$ are determined from one Gröbner basis provided that the set of minimal elements with respect to $\prec_{\mathcal{A}}$ in the set of minimal $\mathcal{A}$-degrees of $I_{\mathcal{A}}$ is known.

As immediate consequences of the above theorem we have the following.

Corollary 14. For each $i \in\{1, \ldots, r\}$, let $\prec_{i}$ denote a degree reverse lexicographical term order on $R$ which has $X_{i}$ as the lowest variable. If a system of generators of $I_{\mathcal{A}}$ is a reduced Gröbner basis with respect to $\prec_{i}$, for every $i \in\{1, \ldots, r\}$, then $I_{\mathcal{A}}$ is generated by its indispensable binomials. 
Observe that from the above corollary and Lemma 8.4 in [19], we get a new proof of the result by I. Peeva and B. Sturmfels in [18] which states that every generic lattice ideal has a unique minimal set of binomial generators.

Let $\mathbf{u}_{+}$and $\mathbf{u}_{-}$denote the positive and negative parts of $\mathbf{u} \in \mathbb{Z}^{r}$, respectively. Given a system of generators $\mathcal{B}$ of $\operatorname{ker}(\mathcal{A}):=\operatorname{ker}\left(\mathbb{Z}^{r} \rightarrow G(\mathcal{A}) ; \mathbf{e}_{i} \mapsto \mathbf{a}_{i}\right)$, we write $I_{\mathcal{B}}$ for the binomial ideal generated by $\mathcal{G}:=\left\{X^{\mathbf{u}_{+}}-X^{\mathbf{u}_{-}} \mid \mathbf{u} \in \mathcal{B}\right\}$. Recall that $I_{\mathcal{B}} \subset I_{\mathcal{A}}$ but $I_{\mathcal{B}} \neq I_{\mathcal{A}}$.

Corollary 15. For each $i \in\{1, \ldots, r\}$, let $\prec_{i}$ denote a degree reverse lexicographical term order on $R$ which has $X_{i}$ as the lowest variable. With the above notation, if $\mathcal{G}$ is a reduced Gröbner basis with respect to $\prec_{i}$, for every $i \in\{1, \ldots, r\}$, then

(a) $I_{\mathcal{B}}=I_{\mathcal{A}}$;

(b) the set of indispensable binomials of $I_{\mathcal{A}}$ is $\mathcal{G}$.

In conclusion, $I_{\mathcal{A}}$ has a unique minimal system of binomial generators.

Proof. (a) By Lemma 12.2 in [22], $\left(I_{\mathcal{B}}:\left(X_{1} \cdots X_{r}\right)^{\infty}\right)=I_{\mathcal{A}}$. Since $\mathcal{G}$ is a reduced Gröbner basis with respect to a degree reverse lexicographical term order on $R$ which has $X_{i}$ as the lowest variable, for each $i \in\{1, \ldots, r\}$, by Theorem 3.1 in [2], it follows that $\left(I_{\mathcal{B}}:\left(X_{1} \cdots X_{r}\right)^{\infty}\right)=I_{\mathcal{B}}$. So, we conclude that $I_{\mathcal{B}}=I_{\mathcal{A}}$.

Now, part (b) is an immediate consequence of Theorem 13 .

We end this section by showing that the Lawrence ideal of $\mathcal{A}$ is generated by indispensable binomials. This result was already proved by P. Pisón-Casares and A. Vigneron-Tenorio in a different context (see Proposition 1(a) in 20]).

Recall that the Lawrence ideal of $\mathcal{A}$ is the ideal of $\mathbb{k}\left[X_{1}, \ldots, X_{r}, Y_{1}, \ldots, Y_{r}\right]$ generated by

$$
\left\{X^{\mathbf{u}} Y^{\mathbf{v}}-X^{\mathbf{v}} Y^{\mathbf{u}}: \sum_{i=1}^{r} u_{i} \mathbf{a}_{i}=\sum_{i=1}^{r} v_{i} \mathbf{a}_{i} \in \mathcal{A}\right\}
$$

Analogously, the Lawrence ideal of $\mathcal{A}$ is the ideal of $\mathbb{k}\left[X_{1}, \ldots, X_{r}, Y_{1}, \ldots, Y_{r}\right]$ associated to the Lawrence lifting of $\mathcal{A}$, that is to say, the ideal associated to the the semigroup generated by $\left(\mathbf{a}_{1}, \mathbf{e}_{1}^{\prime}\right), \ldots,\left(\mathbf{a}_{r}, \mathbf{e}_{r}^{\prime}\right),\left(0, \mathbf{e}_{1}^{\prime}\right), \ldots,\left(0, \mathbf{e}_{r}^{\prime}\right)$ in $G(\mathcal{A}) \oplus \mathbb{Z}^{r}$, where $\mathbf{e}_{i}$ denotes the $i$-th canonical basis vector of $\mathbb{Z}^{r}$.

Corollary 16. The Lawrence ideal of $\mathcal{A}$ is generated by indispensable binomials.

Proof. By [22], Theorem 7.1, any minimal binomial generating set of the Lawrence ideal of $\mathcal{A}$ forms a reduced Gröbner basis, so, by Corollary 14, our claim follows.

Lawrence ideals play a relevant role in the theory of toric and semigroup ideals: on the one hand, Lawrence ideals are the defining ideals toric subvarieties in a product of projective lines $\mathbb{P}^{1} \times \ldots \times \mathbb{P}^{1}$. On the other hand, they are used to compute the Graver basis of $I_{\mathcal{A}}$ (see [22], Chapter 7, for more details).

\section{An eAsy EXAmple}

In this section, we will apply our results to prove that the toric ideal associated to the binary marginal independence model $\mathcal{A}$ induced by the undirected graph $G=$ $(V, E)$ with $V=\{\{1\},\{2\},\{3\},\{4\}\}$ and $E=\{\{1,2\},\{2,3\},\{3,4\},\{4,1\},\{2,4\}\}$ is generated by indispensable binomials.

We refer the interested reader to [10] for the details omitted here about graphical models. 
The model $\mathcal{A}$ has associated matrix

$$
A=\left(\begin{array}{llllllllllllllll}
1 & 0 & 0 & 0 & 0 & 0 & 0 & 0 & 1 & 0 & 0 & 0 & 0 & 0 & 0 & 0 \\
0 & 1 & 0 & 0 & 0 & 0 & 0 & 0 & 0 & 1 & 0 & 0 & 0 & 0 & 0 & 0 \\
0 & 0 & 1 & 0 & 0 & 0 & 0 & 0 & 0 & 0 & 1 & 0 & 0 & 0 & 0 & 0 \\
0 & 0 & 0 & 1 & 0 & 0 & 0 & 0 & 0 & 0 & 0 & 1 & 0 & 0 & 0 & 0 \\
0 & 0 & 0 & 0 & 1 & 0 & 0 & 0 & 0 & 0 & 0 & 0 & 1 & 0 & 0 & 0 \\
0 & 0 & 0 & 0 & 0 & 1 & 0 & 0 & 0 & 0 & 0 & 0 & 0 & 1 & 0 & 0 \\
0 & 0 & 0 & 0 & 0 & 0 & 1 & 0 & 0 & 0 & 0 & 0 & 0 & 0 & 1 & 0 \\
0 & 0 & 0 & 0 & 0 & 0 & 0 & 1 & 0 & 0 & 0 & 0 & 0 & 0 & 0 & 1 \\
1 & 0 & 1 & 0 & 0 & 0 & 0 & 0 & 0 & 0 & 0 & 0 & 0 & 0 & 0 & 0 \\
0 & 1 & 0 & 1 & 0 & 0 & 0 & 0 & 0 & 0 & 0 & 0 & 0 & 0 & 0 & 0 \\
0 & 0 & 0 & 0 & 1 & 0 & 1 & 0 & 0 & 0 & 0 & 0 & 0 & 0 & 0 & 0 \\
0 & 0 & 0 & 0 & 0 & 1 & 0 & 1 & 0 & 0 & 0 & 0 & 0 & 0 & 0 & 0 \\
0 & 0 & 0 & 0 & 0 & 0 & 0 & 0 & 1 & 0 & 1 & 0 & 0 & 0 & 0 & 0 \\
0 & 0 & 0 & 0 & 0 & 0 & 0 & 0 & 0 & 1 & 0 & 1 & 0 & 0 & 0 & 0 \\
0 & 0 & 0 & 0 & 0 & 0 & 0 & 0 & 0 & 0 & 0 & 0 & 1 & 0 & 1 & 0 \\
0 & 0 & 0 & 0 & 0 & 0 & 0 & 0 & 0 & 0 & 0 & 0 & 0 & 1 & 0 & 1
\end{array}\right)
$$

that, in a more condensed form, can be written as

$$
A=\left(\begin{array}{c}
1_{2}^{\prime} \otimes I_{4} \otimes I_{2} \\
I_{4} \otimes 1_{2}^{\prime} \otimes I_{2}
\end{array}\right) \in \mathbb{Z}^{16 \times 16},
$$

where $1_{2}^{\prime}=\left(\begin{array}{ll}1 & 1\end{array}\right), I_{n}$ is the $n \times n$-identity matrix and the symbol $\otimes$ denotes the Kronecker product.

Let $I_{\mathcal{A}} \subset \mathbb{k}\left[X_{1}, \ldots, X_{16}\right]$ be the semigroup ideal associated to the subsemigroup of $\mathbb{N}^{16}$ generated by the columns of $A$. That is to say, $I_{\mathcal{A}}$ is the toric ideal of the independence model $\mathcal{A}$.

The reader may note that by performing row operations on $A$, it becomes a "Lawrence lifting"-type matrix, so, by Corollary 16, we may conclude that $I_{\mathcal{A}}$ is generated by indispensable binomials and hence it has a unique minimal system of binomial generators. Nevertheless, in order to illustrate the results in this paper, we will proceed by assuming that we do not know this fact.

Since $\mathcal{A}$ is a decomposable graphical model, $I_{\mathcal{A}}$ has a quadratic Gröbner basis (see Theorem 4.3 in [10]). Therefore, any indispensable binomial (if it exists) is homogeneous (with the usual grading) and has total degree equal to two. Moreover, every quadratic binomial in $I_{\mathcal{A}}$ consists in differences of square-free monomials, because no sum of two (not necessarily different) columns of $A$ is equal to the double of another one. In conclusion, if $X^{\mathbf{u}}-X^{\mathbf{v}}$ is an indispensable binomial in $I_{\mathcal{A}}$, then $u_{i} \leq 1$ and $v_{i} \leq 1$, for all $i$, where $\mathbf{u}=\left(u_{1}, \ldots, u_{r}\right)^{\prime}$ and $\mathbf{v}=\left(v_{1}, \ldots, v_{r}\right)^{\prime} \in \mathbb{N}^{r}$, as usual.

Thus, the $\mathcal{A}$-degree $\mathbf{a}=A \mathbf{e}$, with $\mathbf{e}=(1, \ldots, 1)^{\prime} \in \mathbb{Z}^{16}$, "captures" all the indispensable binomials.

Now, let us compute the non-negative integer solutions of the system $A \mathbf{u}=\mathbf{a}$. The general solution of the linear system of equation $A \mathbf{u}=\mathbf{a}$ over $\mathbb{Q}$ is

$$
\begin{aligned}
& \mathbf{u}=\left(\begin{array}{lll}
1+a, & 1+b, & 1-a, \quad 1-b,
\end{array}\right. \\
& 1+c, \quad 1+d, \quad 1-c, \quad 1-d, \\
& 1-a, \quad 1-b, \quad 1+a, \quad 1+b, \\
& 1-c, \quad 1-d, \quad 1+c, \quad 1+d)^{\prime}
\end{aligned}
$$

Thus, it is clear that the non-negative integer solutions correspond to the values of $a, b, c$ and $d$ in $\{-1,0,1\}$.

First of all we observe that any $\mathbf{u} \in \mathbb{N}^{16}$ such that $A \mathbf{u}=\mathbf{a}$ has the form

$$
\left(\mathbf{p}_{i}^{\prime}, \mathbf{p}_{j}^{\prime}, \mathbf{p}_{\sigma(i)}^{\prime}, \mathbf{p}_{\sigma(j)}^{\prime}\right)^{\prime}
$$


with

\begin{tabular}{c|cccc|cccc}
$\mathbf{p}_{1}$ & $\mathbf{p}_{2}$ & $\mathbf{p}_{3}$ & $\mathbf{p}_{4}$ & $\mathbf{p}_{5}$ & $\mathbf{p}_{6}$ & $\mathbf{p}_{7}$ & $\mathbf{p}_{8}$ & $\mathbf{p}_{9}$ \\
\hline 1 & 0 & 1 & 2 & 1 & 0 & 2 & 2 & 0 \\
1 & 1 & 0 & 1 & 2 & 0 & 0 & 2 & 2 \\
1 & 2 & 1 & 0 & 1 & 2 & 0 & 0 & 2 \\
1 & 1 & 2 & 1 & 0 & 2 & 2 & 0 & 0
\end{tabular}

and $\sigma=(24)(35)(68)(79)$.

Therefore, it follows that $\nabla_{\mathbf{a}}$ is a direct product of simplicial complexes. Concretely, $\nabla_{\mathbf{a}} \cong K \times K$, with

$$
K=\left\{F \subseteq\left\{Y^{\mathbf{p}_{i}} \mid i=1, \ldots, 9\right\} \mid \operatorname{gcd}(F) \neq 1\right\} .
$$

Furthermore, given $\mathbf{u}=\left(\mathbf{p}_{i}^{\prime}, \mathbf{p}_{j}^{\prime}, \mathbf{p}_{\sigma(i)}^{\prime}, \mathbf{p}_{\sigma(j)}^{\prime}\right)^{\prime}$ and $\mathbf{v}=\left(\mathbf{p}_{k}^{\prime}, \mathbf{p}_{l}^{\prime}, \mathbf{p}_{\sigma(k)}^{\prime}, \mathbf{p}_{\sigma(l)}^{\prime}\right)^{\prime}$, by Corollary 7 and Theorem 8 , we have that $\operatorname{gcd}\left(X^{\mathbf{u}}, X^{\mathbf{v}}\right)^{-1}\left(X^{\mathbf{u}}-X^{\mathbf{v}}\right)$ is indispensable if, and only if,

$$
i=k, j \neq l \text { and } \operatorname{gcd}\left(Y^{\mathbf{p}_{j}}, Y^{\mathbf{p}_{l}}\right) \text { is uniquely attained }
$$

or

$$
i \neq k, j=l \text { and } \operatorname{gcd}\left(Y^{\mathbf{p}_{i}}, Y^{\mathbf{p}_{k}}\right) \text { is uniquely attained. }
$$

In fact, the indispensable binomials are

$$
\operatorname{gcd}\left(Y^{\mathbf{p}_{i}} Z^{\mathbf{p}_{\sigma(\mathbf{i})}}, Y^{\mathbf{p}_{j}} Z^{\left.\mathbf{p}_{\sigma(\mathbf{j})}\right)}\right)^{-1}\left(Y^{\mathbf{p}_{i}} Z^{\mathbf{p}_{\sigma(\mathbf{i})}}-Y^{\mathbf{p}_{j}} Z^{\mathbf{p}_{\sigma(\mathbf{j})}}\right)
$$

with $\operatorname{gcd}\left(Y^{\mathbf{p}_{i}}, Y^{\mathbf{p}_{j}}\right)$ uniquely attained and $Y_{k}=X_{k}, Z_{k}=X_{k+8}, k \in\{1, \ldots, 4\}$ or $Y_{k}=X_{k}, Z_{k}=X_{k+8}, k \in\{5, \ldots, 8\}$.

Summarizing, the indispensable binomials of $I_{\mathcal{A}}$ are determined by the pair of vertices of $K, \mathcal{P}$, whose greatest common divisor is different from the greatest common divisor of any 2-dimensional face of $K$ containing them.

Notice that the natural action of $H=\left\langle\left(\begin{array}{lll}1 & 2 & 3\end{array} 4\right)\right\rangle$ on $\left\{Y_{1}, Y_{2}, Y_{3}, Y_{4}\right\}$ leaves $K$ invariant. In fact, this action is the same as the one given by $\tilde{H}=\left\langle\left(\begin{array}{l}2 \\ 3\end{array} 45\right)\left(\begin{array}{l}6 \\ 7\end{array} 89\right)\right\rangle$ on the set of $\mathbf{p}_{i}$ 's. Thus, in order to compute $\mathcal{P}$, it suffices to perform the computation modulo $H$. There are nine different 1-dimensional faces modulo $H$ :

$$
\begin{gathered}
\left\{Y^{\mathbf{p}_{1}}, Y^{\mathbf{p}_{2}}\right\},\left\{Y^{\mathbf{p}_{1}}, Y^{\mathbf{p}_{6}}\right\},\left\{Y^{\mathbf{p}_{2}}, Y^{\mathbf{p}_{3}}\right\},\left\{Y^{\mathbf{p}_{2}}, Y^{\mathbf{p}_{4}}\right\},\left\{Y^{\mathbf{p}_{2}}, Y^{\mathbf{p}_{\mathbf{6}}}\right\} \\
\left\{Y^{\mathbf{p}_{2}}, Y^{\mathbf{p}_{\mathbf{7}}}\right\},\left\{Y^{\mathbf{p}_{2}}, Y^{\mathbf{p}_{\mathbf{8}}}\right\},\left\{Y^{\mathbf{p}_{2}}, Y^{\mathbf{p}_{\mathbf{9}}}\right\},\left\{Y^{\mathbf{p}_{\mathbf{6}}}, Y^{\mathbf{p}_{\mathbf{7}}}\right\},
\end{gathered}
$$

but only three of them have greatest common divisor different from any $2-$ dimensional face of $K$ :

$$
\left\{Y^{\mathbf{p}_{1}}, Y^{\mathbf{p}_{2}}\right\},\left\{Y^{\mathbf{p}_{2}}, Y^{\mathbf{p}_{6}}\right\},\left\{Y^{\mathbf{p}_{2}}, Y^{\mathbf{p}_{9}}\right\} .
$$

An easy computation shows that

$$
\operatorname{gcd}\left(Y^{\mathbf{p}_{i}}, Y^{\mathbf{p}_{j}}\right)^{-1}\left(Y^{\mathbf{p}_{i}}-Y^{\mathbf{p}_{j}}\right)=\left\{\begin{array}{l} 
\pm\left(Y_{1}-Y_{3}\right) \\
\pm\left(Y_{2}-Y_{4}\right)
\end{array}\right.
$$

when $\operatorname{gcd}\left(Y^{\mathbf{p}_{i}}, Y^{\mathbf{p}_{j}}\right)$ is uniquely attained.

Therefore, by (2), we conclude that there are four indispensable binomials in $I_{\mathcal{A}}:$

$$
X_{1} X_{11}-X_{3} X_{9}, X_{2} X_{12}-X_{4} X_{10}, X_{5} X_{15}-X_{7} X_{13} \quad \text { and } \quad X_{6} X_{16}-X_{8} X_{14} .
$$

Moreover, since these four binomials form a Gröbner basis with respect to any term order on $R$ (because their initial monomials have disjoint support) and their 
exponent vectors generate $\operatorname{ker}(\mathcal{A})$, by Corollary [15] we may assume that $I_{\mathcal{A}}$ is generated by its indispensable binomials; that is to say, the toric ideal associated to the binary marginal independence model $\mathcal{A}$ has a unique minimal system of binomial generators.

\section{ACKNOWLEDGMENT}

We want to warmly thank the anonymous referee for helpful comments and remarks.

\section{REFERENCES}

[1] S. Aoki, A. Takemura, R. Yoshida, Indispensable monomials of toric ideals and Markov bases, J. Symbolic Comput. 43 (2008), no. 6-7, 490-507. MR2406969 (2009c:13065)

[2] A. Bigatti, R. La Scala, L. Robbiano, Computing toric ideals, J. Symbolic Comput. 27 (1999), 351-365. MR 1681344 (2000b:13035)

[3] E. Briales, A. Campillo, C. Marijuán, P. Pisón, Combinatorics of syzygies for semigroup algebra, Collect. Math. 49 (1998), 239-256. MR1677160 (99m:13024)

[4] E. Briales, A. Campillo, C. Marijuán, P. Pisón, Minimal systems of generators for ideals of semigroups, J. Pure Appl. Algebra, 124 (1998), 7-30. MR1600261 (98k:20105)

[5] H. Charalambous, A. Katsabekis, A. Thoma, Minimal systems of binomial generators and the indispensable complex of a toric ideal, Proc. Amer. Math. Soc. 135 (2007), 3443-3451. MR.2336556 (2009a:13033)

[6] H. Charalambous, A. Thoma, On simple $\mathcal{A}$-multigraded minimal resolutions, Contemporary Mathematics, Vol. 502, Amer. Math. Soc., 2009, pp. 33-44.

[7] P. Diaconis, B. Sturmfels, Algebraic algorithms for sampling from conditional distributions, Ann. Statist. 26(1) (1998), 363-397. MR1608156 (99j:62137)

[8] D. Eisenbud, B. Sturmfels, Binomial ideals, Duke Math. J. 84 (1996), no. 1, 1-45. MR:1394747 (97d:13031)

[9] S. Eliahou, Courbes monomiales et algébre de Rees symbolique, PhD Thesis, Université of Genève, 1983

[10] D. Geiger, C. Meek, B. Sturmfels, On the toric algebra of graphical models, Ann. Statist. 34 (2006), no. 3, 1463-1492. MR2278364 (2007m:60035)

[11] J. Herzog, Generators and relations of abelian semigroups and semigroup rings, Manuscripta Math. 3 (1970), 175-193. MR0269762 (42:4657)

[12] E. Miller, B. Sturmfels, Combinatorial Commutative Algebra. Vol. 227 of Graduate Texts in Mathematics. Springer, New York, 2005. MR2110098 (2006d:13001)

[13] H. Ohsugi, T. Hibi, Indispensable binomials of finite graphs, J. Algebra Appl. 4 (2005), no. 4, 421-434. MR2166253 (2006e:13023)

[14] H. Ohsugi, T. Hibi, Toric ideals arising from contingency tables, in Commutative Algebra and Combinatorics. Ramanujan Mathematical Society Lecture Notes Series, Vol. 4, Ramanujan Mathematical Society, Mysore, India, 2007, pp. 91-115. MR2394671(2009c:13070)

[15] I. Ojeda, A. Vigneron-Tenorio, Simplicial complexes and minimal free resolution of monomial algebras. J. Pure Appl. Algebra 214 (2010), no. 6, 850-861.

[16] I. Ojeda, P. Pisón-Casares, On the hull resolution of an affine monomial curve, J. Pure Appl. Algebra 192 (2004), 53-67. MR2067188 (2005e:13018)

[17] I. Ojeda, Examples of generic lattice ideals of codimension 3, Comm. Algebra 36 (2008), 279-287. MR.2381125 (2008j:13027)

[18] I. Peeva, B. Sturmfels, Generic lattice ideals, J. Amer. Math. Soc. 11 (1998), 363-373. MR 1475887 (98i:13022)

[19] I. Peeva, B. Sturmfels, Syzygies of codimension 2 lattice ideals, Math. Z. 229 (1998), 163-194. MR.1649322 (99g:13020)

[20] P. Pisón-Casares, A. Vigneron-Tenorio, On Lawrence semigroups, J. Symbolic Comput. 43 (2008), 804-810. MR2432958 (2009i:20128)

[21] J.C. Rosales, P.A. García-Sánchez, Finitely generated commutative monoids, Nova Science Publishers, Inc., New York, 1999. MR.1694173 (2000d:20074) 
[22] B. Sturmfels, Gröbner bases and convex polytopes, volume 8 of University Lecture Series, American Mathematical Society, Providence, RI, 1996. MR.1363949 (97b:13034)

[23] A. Takemura, S. Aoki, Some characterizations of minimal Markov basis for sampling from discrete conditional distributions, Ann. Inst. Statist. Math. 56(1) (2004), 1-17. MR2053726 (2005g:62103)

Departamento de Matemáticas, Universidad de Extremadura, E-06071 Badajoz, Spain

E-mail address: ojedamc@unex.es

Departamento de Matemáticas, Universidad de Cádiz, E-11405 Jerez de la Frontera, SPAIN

E-mail address: alberto.vigneron@uca.es 